\title{
Describing the potential and detailing the failures of using technology-based methodologies to study school leader time use
}

\author{
Craig Hochbein \\ Lehigh University, College of Education, Pennsylvania, USA
}

Decades of research have demonstrated the critical contribution of school leaders to both school performance and student outcomes. Although researchers have identified the importance of school leaders, they have not specified malleable factors that reliably account for their performance (Reynolds et al., 2014). One of the more promising areas of study has examined the activities and behaviors of school leaders (Grissom, Loeb, \& Master, 2013). For more than a century, scholars have examined how school leaders allot their time to address the demands of their schools (McMurry, 1913; Sebastian, Camburn, \& Spillane, 2018). Despite substantial technological advancements since the first study of school leader time use (SLTU), the means of data collection have remained largely unchanged (Hochbein et al., 2018). To study SLTU, researchers have relied on observation and self-report data collection techniques (Horng, Klasik, \& Loeb, 2010). Although researchers have implemented a variety of research designs to address validity threats stemming from data collection limitations (e.g., May, Huff, \& Goldring, 2012), they have not exploited technological advancements to substantially improve or change the study of SLTU.

Wireless networks, smartphones, activity trackers, biometric sensors, discrete recording devices, Bluetooth beacons, and other technologies exhibit the potential to enhance and alter the study of SLTU. To take advantage of these technological advancements, researchers of SLTU need to understand the benefits of technology-based methodologies, as well as learn from failed attempts at implementing them. The purpose of this paper is to advance the knowledge about implementing technology-based methodologies to study SLTU. To accomplish this purpose, the paper maintains two objectives. First, propose how implementing technology-based methodologies can mitigate systematic validity threats that weaken claims about SLTU. Second, describe failures and setbacks in the development and application of technology-based methodologies to study SLTU. 


\section{Implementing technology to enhance SLTU research}

Throughout the last one hundred years, researchers have attempted to describe how school leaders allot their time (Bates, 1925), as well as identify factors associated with SLTU (Goldring et al., 2008). Despite differences in era and methodology, results from studies of SLTU have supported three common claims. First, the daily and weekly schedules of school leaders have included extensive working hours (Martin \& Willower, 1981), with a substantial amount of activity occurring during non-instructional hours, such as evenings and weekends (Kelly, 1974). Second, the configuration of school leaders' workdays has consisted of brief and unrelated tasks, with days consisting of unconnected activities often lasting less than 10 minutes (Crowson \& Porter-Gehrie, 1980). Finally, school leaders have dedicated more time to activities categorized as managerial, rather than instructional (Grissom, Loeb, \& Mitani, 2015).

Although multiple studies support each of these assertions, systemic methodological limitations weaken the validity of these claims. The common limitations associated with collecting data through observational and self-report methodologies have captured incomplete or inadequate information about SLTU. Specifically, researchers have relied on limited periods of observation, samples of school leaders, and measurements of time use (Mahone et al., 2019). As a result of these three common limitations, researchers have underestimated SLTU, overrepresented the time use of school leaders working in large metropolitan areas, and insufficiently considered alternative metrics of SLTU (Hochbein, Mahone, \& Vanderbeck, 2016).

The first systematic limitation originates from researchers' reliance on observational data collection, which has provided artificial and unrealistic parameters around the time that school leaders engage in school-related work. For example, Grissom, Loeb, and Master (2013) conducted their observations during the instructional hours of the sampled schools, "During each observation, a timer alerted the observer to record information about the principal's activity in 5-minute increments, beginning about 30 minutes prior to the official start of school and ending with the afternoon bell" (p. 434). Yet, Wolcott (1973) highlighted the limitations of his observations between 8AM and 5PM, "Had the observations been extended to hours spent on school business in the evening, the portion of his day spent in meetings would have been even greater..." (p. 89). 
Since the observations of Wolcott (1973), the expectations and responsibilities of school leaders have likely increased the amount of time they work during evenings, weekends, and holidays. For instance, Pollock and Hauseman (2018) reported how email communication extended the workdays of Canadian school leaders. Similarly, Kane and colleagues (2015) indicated the use of video recordings for teacher observations led to American school leaders viewing the lessons during evenings and weekends. To accurately account for SLTU, researchers need to collect data during non-school hours.

A second systemic methodological limitation arises from researchers' sampling of school leaders. Studies that record actual SLTU, rather than using one-time surveys to gather perceptions, often rely on small sample sizes (Kmetz \& Willower, 1982; Spillane \& Zuberi, 2009). For instance, a review of SLTU literature identified just 5 studies with more than 100 participants (Hochbein, Mahone, \& Vanderbeck, 2016). Studies like Peterson (1977), which observed two school leaders during six days, have implemented appropriate qualitative protocols to provide rich description of SLTU. However, the subjects might not accurately represent the experiences of other school leaders, which creates potential external validity threats (Shadish, Cook, \& Campbell, 2002).

When researchers have observed larger samples of school leaders, they often concentrated on a single school district from an urban context (Krug, Scott, \& Ahadi, 1989). Moreover, researchers have relied on a small selection of school districts serving metropolitan areas. For instance, three studies that included more than 50 participants sampled school leaders working in the Miami-Dade County Public Schools (Grissom, Loeb, \& Masters, 2013; Grissom, Loeb, \& Mitani, 2015; Horng, Klasik, \& Loeb, 2010). Similarly, several studies relied on the same sample of school leaders who worked for an unspecified urban district that operated 52 schools (Barnes et al., 2010; Camburn, Spillane, \& Sebastian, 2010; Goldring et al., 2008; May \& Supovitz, 2011; Spillane, Camburn, \& Pareja, 2007). Yet, the majority of schools in the United States do not resemble the organizational structure and student composition of these large metropolitan schools (Hochbein \& Harbour, 2015). Given that context likely contributes to SLTU (May, Huff, \& Goldring 2012), the time use of school leaders working in large metropolitan districts likely varies from their non-urban peers. 
The third systematic limitation stems from the data collection protocols associated with the two primary methodologies of SLTU research. Throughout the history of SLTU research, investigators have relied on observational and self-report methodologies (Camburn, Spillane, \& Sebastian, 2010; Hochbein et al., 2018; Horng, Klasik, \& Loeb, 2010). Both methodologies rely on data input from individuals, either an observer or a participant. This reliance on data input from observers or participants introduces the likelihood of inaccurate or biased responses. For instance, without understanding the rationale for the action of school leaders, observers might misidentify the purpose of SLTU. Similarly, participants might misestimate their time use, as well as unreliably code their activities. These issues with data collection compromise the results of analyses and threaten the validity of claims about SLTU.

Although researchers have implemented a variety of study designs to reduce biased or inaccurate responses (Grissom, Loeb, \& Master, 2013; May, Huff, Goldring, 2012), the reliance on observational and self-report data has constrained the metrics researchers use to study SLTU. For instance, observers have described how time demands create stress for school leaders (Peterson, 1977). Similarly, school leaders have reported perceptions of their stress resulting from time demands (Pollack \& Hauseman, 2018). However, rather than relying on perceptions of stress, researchers could implement technology-based methodologies to continuously monitor metrics related to stress, such as the heart rate of school leaders.

Some researchers have exploited technological advancements to address limitations of SLTU research. For example, Hochbein and colleagues (2018) demonstrated how smartwatches could extend the observation of school leaders into non-instructional hours. The data collection, which occurred for 28 consecutive days between the hours of 8AM and 8PM, resulted in an 85\% response rate from the sample of 11 school leaders. Applying lessons from Hochbein et al. (2018), Mahone and colleagues (2019) relied on notifications sent to smartphones to not only study SLTU across an extended period of time, but also expand sampling. The data collection of Mahone et al. (2019), which occurred during 20 school days between the hours of 7AM and 7PM, resulted in a 74\% response rate from 61 principals working in 20 school districts. Similarly, Vanderbeck and Hochbein (2018) sampled 12 principals working in multiple school districts and compared SLTU results from 422 digital daily logs completed during the summer months (89\% response 
rate) against 97 digital daily logs completed while school was in session (77\% response rate).

These examples demonstrated how the advancement and proliferation of technology has enabled the possibility of addressing critical limitations of studying SLTU. Inexpensive, unobtrusive, robust, and innovative technologies provide researchers the opportunity to extend observational periods, diversify sampling strategies, and expand data collection techniques (Hochbein et al, 2018). However, the application of technology to study SLTU entails challenges for researchers. To seize the opportunities provided by technological advancements, SLTU researchers need to understand the issues and limitations associated with prior attempts at implementing technology-based methodology.

\section{The trials of implementing technology to alter SLTU research}

Although researchers have implemented technology-based methodologies to enhance the study of SLTU (Hochbein et al., 2018; Sebastian, Camburn, \& Spillane, 2018), they have not altered typical SLTU research methodologies. The use of smartphones and other wireless devices facilitated the extension of observational periods and expansion of sampling, but continued to rely on individuals' report of data (López et al., 2012; Mahone et al., 2018). Activity trackers, Bluetooth sensors, video recorders, and other devices exhibit the potential to alter SLTU research by collecting data that is not reported by observers or participants (Lauer, 2015). To capitalize upon such burgeoning technologies, SLTU researchers must not only recognize potential applications, but also design successful implementation protocols.

From conducting technology-based studies to enhance SLTU research, I realized the potential to implement various technologies that could provide continuous and passive data collection. The capacity to continuously and passively collect data relevant to SLTU presented numerous possibilities to alter SLTU research. For example, activity trackers could collect physiological metrics of stress, rather than observers' or participants' perceptions. Similarly, tracking school leaders' movement through a school presents a potential and promising application of how technology-based methodology could alter SLTU research. 


\subsection{Tracking movements}

Monitoring school leaders' movement throughout a school building could provide knowledge that impacts the preparation and practice of school leaders. Connecting continuous movement data with existing datasets could examine if school leaders exhibit bias in the amount of time they dedicate to certain groups of educators or students. Certainly, trained observers could collect similar data by following school leaders, but observer bias and study expense limit such studies. Although participants could inexpensively report their movements, the data would again be prone to bias, as well as not exist as a continuous measure. In contrast, technological advancements demonstrated the possibilities of inexpensively, passively, continuously, and accurately collecting data about the location of school leaders within their schools.

Numerous technologies exhibited the potential to monitor school leaders' movement throughout their buildings, but critical limitations precluded effective implementation. For instance, Global Positioning System (GPS) technologies could not reliably distinguish between the rooms or floors of a school building. Near Field Communication (NFC) technologies could distinguish between rooms, but their limited communication range (typically a few centimeters) reduces reliability because school leaders needed to actively engage with the communication devices. To address issues of accuracy and reliability, Radio-Frequency Identification (RFID) technologies could monitor school leaders as they pass between the rooms of a school. However, the current expense of implementing a passive RFID system within a school is prohibitive.

These issues of accuracy, reliability, and cost precluded our application of GPS, NFC, and RFID to the study of SLTU. However, the fundamental premise of monitoring continuous communication between two devices led to the consideration of alternative technological solutions. Although a variety of technologies exhibited the potential to track the movement of school leaders within a building, I worked with research teams to develop the implementation of two promising technologies. One research team focused on the use of existing school wireless networks and a second team attempted to implement Bluetooth beacon technology. 


\subsection{Wireless networks}

Many American schools have been equipped with wireless networks that support internet-enabled devices used by educators and students. Schools' wireless networks typically rely on the strategic positioning of wireless routers to secure fast and reliable internet usage. In theory, recording the access of routers by a wireless device would provide researchers the opportunity to monitor the device as it traversed the wireless network. To take advantage of these existing and prevalent technologies, I worked with a group of students to develop a smartphone application, which would record data related to wireless router access. During the course of designing and testing the data collection, we encountered several flawed assumptions that inhibited implementation, but provided lessons for the use of technology-based methodology.

Specifically, we identified issues related to the use of devices that would connect to the wireless networks. We anticipated relying on the smartphones that school leaders already carried and registered on the schools' wireless networks. However, a software application that continuously captured data drained the battery of the devices, which inhibited school leaders' participation. In addition, variation between smartphones created data collection issues. For instance, differences between smartphone platforms and model generations required the design of multiple software options. More importantly, differences between smartphone models resulted in variance in accessing wireless routers. Some models maintained wireless access for as long as possible, whereas other models immediately switched to the strongest available wireless signal.

Although use of a standard smartphone model supplied to participants could reduce these issues, assumptions about schools' wireless networks proved more challenging. Schools' wireless networks typically relied on the deployment of a multitude of wireless routers, often resulting in a classroomdedicated router. However, to ensure coverage for the entire school, the wireless radius of the classroom routers routinely overlapped. As a result, analyses of access to wireless routers would suffer from issues of accuracy and reliability. Working with school personnel to alter the radiuses of wireless routers or creating unique analytic models for each school could mitigate these validity threats. However, such adjustments were neither practical, nor efficient. Together, these issues precluded our ability to capitalize on the prevalence of technology that already existed within schools. 


\subsection{Bluetooth beacons}

Recognizing the impediments of relying on the existing technologies within schools, I worked with a research team to develop a network of beacons that relied on Bluetooth technology to interact with Bluetooth-enabled devices. Several manufacturers offered affordable Bluetooth beacons that supported the development of a network. In contrast to the use of the existing wireless networks, developing a network of beacons would create a standardized and independent communication system between beacons and devices. Moreover, the beacons would enable the development of a system that could be reused and adapted to school sites.

Although beacons enabled the development of a standardized, reliable, and affordable network, the precision of tracking device access across the network continued to impede implementation for researching school leader movements. As the beacons were constructed to communicate with, but not track devices, additional software was needed to capture the communication data. In developing the software, the research team again encountered the issue of a device communicating with multiple beacons. However, the beacons provided two advantages over existing wireless networks.

First, researchers could adjust the beacons' radiuses, which would limit substantial overlap between them. As the beacons did not influence the instructional activities of a school, researchers could more readily tinker with the radiuses of the beacons. Second, devices could communicate with multiple beacons simultaneously, capturing the strength of the signal as a continuous metric. Theoretically, the metrics from multiple devices would enable triangulation of the position of a device and thus the school leader carrying it. To actualize this concept, the research team has continued to develop and test systematic protocols for strategically placing the beacons to build the network, as well as develop the analytic models that calculate school leaders' position and movement within a school. However, we have yet to implement a beacon network to study SLTU.

\section{Lessons of implementing technology-based methodology to study SLTU}

These setbacks and failed attempts to monitor school leader movement provided lessons and guidance for future efforts of implementing technology-based methodologies. For researchers of SLTU, the efforts 
elucidate several discipline-specific recommendations. These attempts to examine SLTU in a novel manner demonstrated the need for researchers to monitor and consider how technological advancements could address common research limitations. For instance, SLTU researchers might explore how school leaders' currentinteractions with technology can be used to collect time use data. Electronic communications, social media usage, school video cameras, and other pervasive technologies present potential data collection opportunities. However, researchers need to ensure that capitalizing on existing technology does not interrupt school leaders' typical duties or interfere with the instructional activity of a school. Relatedly, as technologies enable more extensive and precise monitoring of SLTU, researchers should also engage in ethical considerations of the impact of such studies on the personal and professional experiences of the participants.

In addition to the specific considerations for researchers studying SLTU, the described efforts offered some guidance for researchers from other disciplines who wish to implement technology-based methodologies. The pervasiveness of technologies capable of collecting pertinent data presents researchers with unprecedented opportunities. However, many common or popular devices were not produced for the purpose of conducting research. Despite the theoretical potential of common technologies, the data collection, storage, or extraction might not satisfy the standards or needs of researchers. For example, some researchers might be able to utilize popular activity trackers for their studies, whereas others might require the purchase of more expensive devices that are designed specifically for research-use. As a result of such discrepancies, researchers need to identify and test their assumptions about the capacity of technology to satisfy their needs. Small-scale pilot testing can help researchers ascertain if the technology can accomplish the intended research objectives.

The proliferation and pervasiveness of technology has created opportunities for scholars to conduct unprecedented research. For instance, technological advancements demonstrate the potential for SLTU researchers to address systematic limitations of the existing literature. Unfortunately, the theoretical possibilities of implementing technology-based methodologies do not always match the experiences of researchers. As setbacks and failures in the implementation of technology-based methodologies do not typically result in scholarly publications, researchers need to find ways to disseminate lessons and recommendations from their attempts. Reporting unsuccessful 
endeavors can increase the likelihood of successfully implementing technology-based methodologies by preventing researchers from repeating avoidable mistakes.

\section{References}

Barnes, C. A., Camburn, E., Sanders, B. R., \& Sebastian, J. (2010). Developing instructional leaders: Using mixed methods to explore the black box of planned change in principals' professional practice. Educational Administration Quarterly, 46(2), 241-279.

Bates, G. (1925). Functions of the elementary school principal. Journal of Educational Method, 4,178-184.

Camburn, E. M., Spillane, J. P., \& Sebastian, J. (2010). Assessing the utility of a daily log for measuring principal leadership practice. Educational Administration Quarterly, 46(5), 707-737.

Crowson, R. L. \& Porter-Gehrie (1980). The discretionary behavior of principals in large-city schools. Educational Administration Quarterly, 16(1), 45-69.

Goldring, E., Huff, J., May, H., \& Camburn, E. (2008). School context and individual characteristics: What influences principal practice? Journal of Educational Administration, 46(3), 332-352.

Grissom, J. A., Loeb, S., \& Master, B. (2013). Effective instructional time use for school leaders: Longitudinal evidence from observations of principals. Educational Researcher, 42(8), 433-444.

Grissom, J. A., Loeb, S., \& Mitani, H. (2015). Principal time management skills: Explaining patterns in principals' time use, job stress, and perceived effectiveness. Journal of Educational Administration, 53(6), 773-793.

Hochbein, C., Dever, B. V., White, G., Mayger, L. K., \& Gallagher, E. (2018). Confronting methodological challenges in studying school leader time use through technological advancements: A pilot study. Educational Management Administration \& Leadership, 46(4), 659-678.

Hochbein, C. \& Harbour, K. E. (2015). Large and small urban school districts: Empirical identification and comparison utilizing student population. In K. L. Sanzo, I. Sutherland, \& J. Scribner (Eds.), Leading small and mid-sized urban school districts (pp. 1-19). Bingley, United Kingdom: Emerald Group Publishing.

Hochbein, C., Mahone, A. S., \& Vanderbeck, S. C. (2016). A systematic review of principal time use research sampling and observation periods. Paper presented at the annual conference of the University Council for Educational Administration, Detroit, MI.

Horng, E. L., Klasik, D., \& Loeb, S. (2010). Principal's time use and school effectiveness. American Journal of Education, 116(4), 491-523.

Kane, T., Gehlbach, H., Greenberg, M., Quinn, D., \& Thal, D. (2015). The Best Foot Forward Project: Substituting teacher-collected video for in-person classroom observations. First year implementation report. Cambridge, MA: Center for Education Policy Research, Harvard University.

Kelly, N. T. (1974). An ethnography of a secondary school principal. Unpublished doctoral dissertation. New Mexico State University. 
Kmetz, J. T. \& Willower, D. J. (1982). Elementary school principals' work behavior. Educational Administration Quarterly, 18(4), 62-78.

Krug, S. E., Scott, C., \& Ahadi, S. (1989). An experience sampling approach to the study of principal instructional leadership 1: Results from the principal activity sampling form. Urbana, IL: National Center for School Leadership.

Lauer, T. (2015). Tech solutions to principals' overloaded schedules. Education Week. 35(11), 20. López, V., Ahumada, L., Galdames, S., \& Madrid, R. (2012). School principals at their lonely work: Recording workday practices through ESM logs. Computers \& Education, 58(1), 413-422.

Mahone, A. S., Hochbein, C., Dever, B. D., \& Farley-Ripple, E. N. (2019). Within-principal variation: Exploring the relationships between time use and school context. Paper presented at the annual conference of the American Educational Research Association, Toronto, Canada.

Martin, W. J., \& Willower, D. J. (1981). The managerial behavior of high school principals. Educational Administration Quarterly, 17(1), 69-90.

May, H., Huff, J., \& Goldring, E. (2012). A longitudinal study of principals' activities and student performance. School Effectiveness and School Improvement, 23(4), 417-439.

May, H., \& Supovitz, J. A. (2011). The scope of principal efforts to improve instruction. Educational Administration Quarterly, 47, 332-352.

McMurry, F. M. (1913). Elementary School Standards. New York: World Book Company.

Peterson, K. D. (1977). The principal's tasks. Administrator's Notebook, 26(8), 2-5.

Pollock, K., \& Hauseman, D. C. (2018). The Use of e-mail and principals' work: A double-edged sword. Leadership and Policy in Schools, 1-12.

Reynolds, D., Sammons, P., De Fraine, B., Van Damme, J., Townsend, T., Teddlie, C., \& Stringfield, S. (2014). Educational effectiveness research: A state-of-the-art review. School Effectiveness and School Improvement, 25(2), 197-230.

Sebastian, J., Camburn, E. M., \& Spillane, J. P. (2018). Portraits of principal practice: Time allocation and school principal work. Educational Administration Quarterly, 54(1), 47-84.

Shadish, W. R., Cook, T. D., \& Campbell, D. T. (2002). Experimental and quasi-experimental designs for generalized causal inference. New York, NY: Houghton Mifflin.

Spillane, J. P., Camburn, E. M., \& Pareja, A. (2007). Taking a distributed perspective to the school principal's workday. Leadership and policy in schools, 6(1), 103-125.

Spillane, J. P., \& Zuberi, A. (2009). Designing and piloting a leadership daily practice log: Using logs to study the practice of leadership. Educational Administration Quarterly, 45, 375-423.

Vanderbeck, S. C. \& Hochbein, C. (2018). The content and configuration of principal time use during the summer and fall. Paper presented at the annual conference of the University Council for Educational Administration, Houston, TX.

Wolcott, H. F. (1973). The man in the principal's office: An ethnography. New York: Holt, Rinehart and Winston.

\section{Author}

Dr. Craig Hochbein, Lehigh University, College of Education, Iacocca Hall, 111 Research Drive Bethlehem, PA 18015, USA, e-mail: crh313@lehigh.edu 